\title{
Stroke in long-term intensive care unit
}

\author{
(D) Gülhan Sarıçam \\ Pursaklar State Hospital, Department of Neurology, Ankara, Turkey
}

Cite this article as: Sarıçam G. Stroke in long-term intensive care unit. J Health Sci Med 2021; 4(2): 118-122.

\begin{abstract}
Aim: Long-term acute care hospitals (LTACH) provide specialized care for patients recovering from severe acute diseases and for chronic and critically ill patients; who need long-term ventilatory support. This study aimed to investigate the factors affecting the length of stay of stroke patients in our long-term intensive care unit (LTICU).

Material and Method: This retrospective study included 200 stroke patients; who were followed up in the LTICU. Demographic characteristics, comorbid diseases, and culture results of patients were examined as variables and the effects of these variables on the length of intensive care unit stay were analyzed.

Result: Of the patients; the mean age was 79 (41-99) years, 99 (49.5\%) were males, and the mean length of stay was 46 (7-463) days. The length of stay in LTICU was significantly long in stroke patients with a tracheostomy and positive growth in urine cultures $(\mathrm{p}=0.013, \mathrm{p}=0.018)$. The length of stay was significantly short in patients; who received total parenteral nutrition $(\mathrm{TPN})(\mathrm{p}<0.01)$.

Conclusion: Our study has demonstrated that presence of a tracheostomy and positive growth in urine cultures increased but TPN significantly decreased the length of stay of stroke patients in LTICU. To optimize LTACH facilities for stroke patients; we suggest that the overall pattern of care in such centers should be better known, further integration with acute care units should be established, and further studies should be conducted.
\end{abstract}

Keywords: Long-term intensive care unit, stroke, tracheostomy, urine cultures

\section{INTRODUCTION}

Long-term acute care hospitals (LTACH) provide specialized care for patients recovering from severe acute diseases and for patients with chronic and critical illnesses; who need long-term ventilatory support $(1,2)$. Stroke is the main cause of severe disability. Despite advances in prevention and treatment, stroke has been the second most common cause of death for the last two decades (3). However, the percentage of stroke patients admitted to intensive care units for monitorization and management of post-stroke complications continues to grow (4).

Chronic and critical diseases such as stroke place a huge burden on the healthcare system (5). Clinicians; who wish to develop innovative care strategies for such patients, transfer these patients from traditional short-term care hospitals to LTACH (1). The Centers for Medicare and Medicaid Services describe LTACH as acute care facilities with a mean length of hospital stay of 25 days or longer (6). Typically; LTACH provide care to patients, who do not need full-fledged services of a short-term care facility but who need ongoing care considerably (7).
Despite the growing role of long-term acute care, population-based data about overall patterns of use of LTACH is very limited and little is known about the evolution of the characteristics of patients transferred to long-term acute care. In the literature, no studies are available about long-term intensive care of stroke patients. The aim of this study was to evaluate the demographic characteristics, comorbid diseases, and culture test results of stroke patients and to analyze the effects of these factors on the length of stay in the longterm intensive care unit (LTICU).

\section{MATERIAL AND METHOD}

The study was approved by Ankara Numune Training and Research Hospital's Ethics Committee (date: September 06, 2018; Approval No: E-18-2200). All study procedures were conducted in compliance with the principles of the Declaration of Helsinki. Files of 200 patients; who were followed up in LTICU for stroke in the period from January 1, 2015 to January 1, 2020, 
were reviewed retrospectively. Stroke patients were categorized into the ischemic stroke (IS), intracerebral hematoma ( $\mathrm{ICH})$, and subarachnoid hemorrhage (SAH) groups. Age, gender, Glasgow Coma Scale (GCS) scores, the Acute Physiology and Chronic Health Evaluation II (APACHE II) scores of the study patients; percentages of mechanical ventilation (MV) use, tracheostomies, percutaneous endoscopic gastrostomies (PEG), pressure ulcers (PU), enteral nutrition (EN) (via PEG and nasogastric tube), and total parenteral nutrition (TPN) use; comorbid diseases including hypertension (HT), diabetes mellitus (DM), heart disease (HD), and the effects of these variables on the length of intensive care unit stay were investigated. In LTICU, stroke patients were primarily tried to be fed enterally. TPN was used when EN was not possible (GIS problems, PEG infections, etc). Calorie and protein needs were calculated for each patient by taking age, height, weight, gender, and body temperature into account. The culture test results of the study patients in the blood, urine, wound, rectal, and tracheal aspirate samples, and the effects of these culture test results on the length of intensive care unit stay were examined. Bacteria growing in culture samples were grouped as Escherichia coli (E. coli), Methicillin-resistant Staphylococcus aureus (MRSA), Acinetobacter spp., Pseudomonas spp., Proteus spp., and other bacterial spp (Enterococcus, Candida spp., etc.).

GCS comprises criteria to assess the general neurological status including the level of consciousness of the patient. GCS scores are determined based on the verbal response, motor response, and eye-opening response of the patient. A score of 15 points is assigned for the best response and a score of 3 points is assigned for the worst response (8).Total APACHE II scores can range from 0 to 71, with increasing scores representing higher disease severity (9).

\section{Statistical Analysis}

The study data collected from 200 patients were transferred to the IBM SPSS Statistics 23 package software for statistical analyses. Categorical data were presented via frequency distributions (in numbers and percentages). Numerical data were summarized by descriptive statistics (mean, standard deviation, median, and minimum and maximum values). The numerical variables were tested by the KolmogorovSmirnov normality test; which revealed a non-normal distribution $(\mathrm{p}<0.05)$. Therefore, non-parametric statistical tests were used in the statistical analysis of the study data. Differences between two independent groups were tested by the Mann-Whitney $U$ test. Differences across more than two groups were analyzed by the Kruskal-Wallis test.

\section{RESULTS}

Of 217 patients followed up in LTICU in the period from 2015 to 2020, 17 were excluded from the study because of missing data and recurrent admissions. Therefore, a total of 200 patients were included in the study for statistical analysis. Ninety-nine (49.5\%) patients were men; 101 (50.5\%) patients were women, and the mean age of the patients was 79 (41-99) years. While 43 (21.5\%) patients were discharged, 157 (78.5\%) died. The mean GCS and APACHE-II scores were 7 and 24, respectively. The mean length of stay was 46 (7-463) days (Table 1).

\begin{tabular}{|c|c|c|}
\hline Variables & Median & Min.-Max. \\
\hline Age (years) & 79.0 & $41-99$ \\
\hline GCS & 7.0 & $3-12$ \\
\hline APACHE II & 24.0 & $12-40$ \\
\hline \multirow{2}{*}{ LOS in LTICU (days) } & 46.0 & $7-463$ \\
\hline & $\mathrm{N}$ & $\%$ \\
\hline \multicolumn{3}{|l|}{ Gender } \\
\hline Female & 101 & 50.5 \\
\hline Male & 99 & 49.5 \\
\hline
\end{tabular}

The diagnoses were IS in $134(67 \%)$ patients, ICH in $42(21 \%)$ patients, and SAH in $24(12 \%)$ patients. HT was present in 194 (97\%) patients; HD was present in $99(49.5 \%)$ patients, and DM was present in $49(24.5 \%)$ patients (Table 2 ).

\begin{tabular}{|lcc|}
\hline \multicolumn{3}{l}{ Table 2. Patient conditions/comorbidities } \\
\hline Conditions/comorbidities & $\mathbf{N}$ & $\%$ \\
\hline IS & 134 & 67.0 \\
ICH & 42 & 21.0 \\
SAH & 24 & 12.0 \\
HT & & \\
Present & 194 & 97.0 \\
Absent & 6 & 3.0 \\
DM & & \\
Present & 49 & 24.5 \\
Absent & 151 & 75.5 \\
HD & & \\
Present & 99 & 49.5 \\
Absent & 101 & 50.5 \\
\hline IS: Ischemic stroke, ICH: Intracerebral hematoma, SAH: Subarachnoid hemorrhage, \\
HT: Hypertension, DM: Diabetes mellitus, HD: Heart disease \\
\hline \multicolumn{2}{l}{} \\
\hline
\end{tabular}

Mechanical ventilation support was provided to 162 (81\%) patients. A tracheostomy and a PEG were present in $172(86 \%)$ and $174(87 \%)$ patients, respectively. Eightyfive (42.5\%) patients were administered TPN and 196 (98\%) patients received EN (PEG and nasogastric tube). A PU was found in 167 (83.5\%) patients (Table 3). 


\begin{tabular}{|lcc|}
\hline Table 3. Patient characteristics & & \\
\hline Concomitant problems & N & $\%$ \\
\hline MV & 162 & 81.0 \\
Present & 38 & 19.0 \\
Absent & & \\
Tracheostomy & 172 & 86.0 \\
Present & 28 & 14.0 \\
Absent & & \\
PEG & 174 & 87.0 \\
Present & 26 & 13.0 \\
Absent & & \\
EN & 196 & 98.0 \\
Present & 4 & 2.0 \\
Absent & & \\
NG & 22 & 11.0 \\
Present & 178 & 89.0 \\
Absent & & \\
PU & 167 & 83.5 \\
Present & 33 & 16.5 \\
Absent & & \\
TPN & 85 & 57.5 \\
Present & 115 & \\
Absent & & \\
Discharge Condition & 43 & \\
Alive & 157 & \\
Exitus & & \\
\hline MV: Mechanical ventilation, PEG: Percutaneous endoscopic gastrostomy, EN: Enteral \\
nutrition, NG: nasogastric tube TPN: Total parenteral nutrition, PU: Pressure ulcer
\end{tabular}

When culture test results were examined, no statistically significant differences were observed in the length of LTICU stay by the species of bacteria isolated from culture samples $(\mathrm{p}>0.05)$. Methicillin Resistant Staphylococcus aureus (MRSA) and E. coli growth occurred in the blood culture tests of 85 (42.5\%) and 23 (11.5\%) patients, respectively. E. coli and Pseudomonas growth occurred in urine culture tests of $57(28.5 \%)$ and $16(8 \%)$ patients; in wound culture tests of $30(15 \%)$ and $20(10 \%)$ patients, and in tracheal culture tests of $22(11 \%)$ and 18 (9\%) patients, respectively (Table 4).

The length of LTICU stay was statistically significantly different by the presence of a tracheostomy, TPN use, and bacterial growth in urine culture tests $(\mathrm{p}<0.05)$. The length of LTICU stay was significantly longer in patients with a tracheostomy and in patients with positive bacterial growth in urine culture tests ( $\mathrm{p}=0.013$ and $\mathrm{p}=0.018$, respectively). The length of LTICU stay was significantly shorter in the patients; who received TPN $(\mathrm{p}<0.01)$. However; age, gender, GCS or APACHE II scores, diagnosis; presence of comorbidities, MV, PEG, or PU, and the culture test results excluding urine cultures did not act on the length of LTICU stay (Table 5).
Table 4. Examination of the relationship between the length of stay and the bacteria type

\begin{tabular}{|c|c|c|c|c|}
\hline Type of Culture & $\mathbf{N}$ & $\begin{array}{c}\text { Length of Stay } \\
\text { Median (Min.-Max.) }\end{array}$ & K.W. & $\mathbf{P}$ \\
\hline \multicolumn{5}{|l|}{ Blood } \\
\hline E. coli & 23 & $53(10-280)$ & \multirow{4}{*}{1.016} & \multirow{4}{*}{0.797} \\
\hline Acinetobacter spp. & 16 & $59.5(11-305)$ & & \\
\hline$M R S A$ & 85 & 45 (7-249) & & \\
\hline Others & 11 & $36(13-247)$ & & \\
\hline \multicolumn{5}{|l|}{ Urine } \\
\hline E. coli & 57 & $44(10-463)$ & \multirow{5}{*}{3.138} & \multirow{5}{*}{0.535} \\
\hline Pseudomonas spp. & 16 & $61.5(12-142)$ & & \\
\hline Acinetobacter spp. & 8 & $70(13-247)$ & & \\
\hline Proteus spp. & 7 & $90(10-280)$ & & \\
\hline Others & 8 & $118(12-198)$ & & \\
\hline \multicolumn{5}{|l|}{ Wound } \\
\hline E. coli & 30 & $42(9-375)$ & \multirow{5}{*}{3.637} & \multirow{5}{*}{0.457} \\
\hline Pseudomonas spp. & 20 & $52.5(13-305)$ & & \\
\hline Acinetobacter spp. & 15 & $60(17-283)$ & & \\
\hline$M R S A$ & 10 & $53.5(41-249)$ & & \\
\hline Others & 9 & $36(10-280)$ & & \\
\hline \multicolumn{5}{|l|}{ Tracheal Aspirate } \\
\hline E. coli & 22 & $43(9-164)$ & \multirow{4}{*}{2.093} & \multirow{4}{*}{0.553} \\
\hline Pseudomonas spp. & 18 & $64.5(12-463)$ & & \\
\hline Acinetobacter spp. & 17 & $44(10-247)$ & & \\
\hline Others & 13 & $62(10-280)$ & & \\
\hline
\end{tabular}

Table 5. Variables related to the length of stay in a long-term intensive care unit

\begin{tabular}{|c|c|c|c|c|}
\hline & $\mathbf{N}$ & $\begin{array}{c}\text { Length of Stay } \\
\text { Median (Min.-Max.) }\end{array}$ & $\mathrm{Z}$ & $\mathbf{P}$ \\
\hline \multicolumn{5}{|l|}{ Tracheostomy } \\
\hline Present & 172 & $48.5(7-463)$ & \multirow{2}{*}{-2.477} & \multirow{2}{*}{$0.013^{*}$} \\
\hline Absent & 28 & $33.5(10-375)$ & & \\
\hline \multicolumn{5}{|l|}{ TPN } \\
\hline Present & 85 & $43(9-305)$ & \multirow{2}{*}{-2.768} & \multirow{2}{*}{$0.006^{*}$} \\
\hline Absent & 115 & $53(7-463)$ & & \\
\hline \multicolumn{5}{|l|}{ Urine Culture } \\
\hline Presence of growth & 96 & $54.5(10-463)$ & \multirow{2}{*}{-2.356} & \multirow{2}{*}{$0.018^{\star}$} \\
\hline No growth & 104 & $44.5(7-375)$ & & \\
\hline
\end{tabular}

\section{DISCUSSION}

Acute stroke is one of the leading reasons for admission to neurological ICUs. Minor progress that was achieved so far in stroke management approaches continues to be the cause of current high mortality and morbidity rates $(10,11)$. Stroke patients are first seen in an emergency room then admitted to an acute care hospital in an average of 4-5 days. This process is followed by admission to an inpatient rehabilitation facility, a long-term acute care hospital (LTACH), a skilled nursing facility, home healthcare, outpatient 
clinic care, or a combination of such facilities (12). Acute stroke patients may need a long time of hospital stay due to a need for MV, intravenous antibiotic therapy, or wound care (13). Of the 200 stroke patients admitted to our LTICU from acute ICUs; 134 (67\%) had a diagnosis of IS, 42 (21\%) had ICH, and $24(12 \%)$ had SAH. Of these patients; $81 \%$ needed MV and $83.5 \%$ had PU requiring care.

Kahn et al. (14) have demonstrated that mortality rates range from $8.4 \%$ to $48.1 \%$ in LTACH. Scheinhorn et al. (2) observed in their multi-center study that the mean age was 71.8 years and the median length of stay was 40 days in LTACH patients, and one in three survived 12 months after admission. We found out that the mean age was 79 years, the median length of stay was 46 days, and the mortality rate was $78.5 \%$ in our stroke patients followed up in LTICU. We have found no similar studies in the literature on stroke patients treated in LTICU.

A tracheostomy is a common procedure used in patients with acute respiratory distress, especially when clinicians predict the need for long-term MV in a patient (15). Tracheostomy use in stroke patients has increased steadily over the past two decades. Chatterjee et al. (16) observed that increased use of tracheostomy in stroke patients was associated with earlier placement, reduced in-hospital mortality, and a shorter average length of stay. However; they demonstrated that discharge rates to non-acute care centers increased but rates of discharge to home decreased in stroke patients, who underwent a tracheostomy procedure. Consistent with that study; a tracheostomy was present in $86 \%$ of the stroke patients admitted to our hospital's LTICU in our study. However; we found out that the length of stay in the LTICU was significantly longer in stroke patients with a tracheostomy contrary to that of acute-care ICUs. Similarly; Turcotte et al. (17) demonstrated an increased length of stay in LTACH patients, who need dialysis, tracheostomy care, ventilator support, and the placement of a feeding tube.

Artificial nutrition support has become the primary therapeutic intervention for preventing metabolic impairments and loss of lean body mass in order to improve outcomes in critically ill patients (18). Although enteral feeding is physiological and is beneficial for the protection of microbial versatility and the structural and functional integrity of the intestines, the use of the enteral route for nutrition is associated with disadvantages especially in acute disease stages and gastrointestinal dysfunction $(19,20)$. Almost all patients $(98 \%)$ followed up in LTICU received EN in our study but $85(42.5 \%)$ patients received TPN due to gastrointestinal problems and issues with PEG. We have found out in our study that the length of stay in the LTICU was significantly shorter in stroke patients; who received TPN support, compared to the other patients. It has been shown that TPN is a favorable source for protein and calorie support when $\mathrm{EN}$ is not possible or used as a supplementary method $(21,22)$.

Healthcare-associated infections constitute an important health problem and act on morbidity and mortality significantly (23). Bogason et al. (24) demonstrated that urinary tract infections of any type were associated with an increased length of stay in hospitalized stroke patients. Similar to the findings of the current study; it has been reported that infections resistant to antibiotics and HIV infections increased the length of stay in long-term care hospitals (17). Consistent with the findings of that study, we have found out in our study that the length of stay was significantly long in patients with positive growth in urine culturww

\section{CONCLUSION}

We observed that the presence of a tracheostomy and positive growth in urine cultures increased but TPN administration reduced the length of stay of stroke patients followed up in LTICU. In parallel with the aging population and advances in critical care, LTACHs are becoming increasingly and significantly involved in the provision of care for critical chronic diseases including stroke. We think that further information is needed about the overall LTACH use patterns of stroke patients; that integration with acute care units should be improved, and that further studies are required in order to optimize the use of LTACH by stroke patients.

\section{ETHICAL DECLARATIONS}

Ethics Committee Approval: The study was carried out with the permission of the Research Ethics Committee of Ankara Numune Training and Research Hospital (date: September 06, 2018; Approval No: E-18-2200)

Informed Consent: Because the study design was retrospectively, no written informed consent form was obtained from patients.

Referee Evaluation Process: Externally peer-reviewed.

Conflict of Interest Statement: The authors have no conflicts of interest to declare.

Financial Disclosure: The authors declare that this study has received no financial support.

Author Contributions: All of the authors declare that they all participated in the design and the conduct of the study, and in the analysis of the study data. All authors have approved the final version. 


\section{REFERENCES}

1. Liu K, Baseggio C, Wissoker D, Maxwell S, Haley J, Long S. Longterm care hospitals under Medicare: facility-level characteristics. Health Care Financ Rev 2001; 23: 1-18.

2. Scheinhorn DJ, Hassenpflug MS, Votto JJ, et al. Post-ICU mechanical ventilation at 23 long-term care hospitals: a multicenter outcomes study. Chest 2007; 131: 85-93.

3. Donnan GA, Fisher M, Macleod M, Davis SM. Stroke. Lancet 2008; 371: 1612-23.

4. Kirkman MA, Citerio G, Smith M. The intensive care management of acute ischemic stroke: an overview. Intensive Care Med 2014; 40: 640-53.

5. Kahn JM, Le T, Angus DC, et al. The epidemiology of chronic critical illness in the United States 2015; 43: 282-7.

6. Carson SS. Know your long-term care hospital. Chest 2007; 131: $2-5$.

7. Scheinhorn DJ, Hassenpflug MS, Votto JJ, et al. Ventilatordependent survivors of catastrophic illness transferred to 23 long-term care hospitals for weaning from prolonged mechanical ventilation. Chest 2007; 131: 76-84.

8. Teasdale G, Jennett B. Assessment of coma and impaired consciousness. A practical scale. Lancet 1974; 2: 81-4.

9. Knaus WA, Draper EA, Wagner DP, Zimmerman JE. APACHE II a severity of disease classification system. Critical care medicine 1985; 13: 818-29.

10. Backhaus R, Aigner F, Schlachetzki F, et al. Inventory of a neurological intensive care unit: who is treated and how long? Neurol Res Int 2015; 2015: 696038.

11. Vincent JL, Singer M, Marini JJ, et al. Thirty years of critical care medicine. Crit Care 2010; 14: 311.

12. Cramer SC, Wolf SL, Adams HP, et al. Stroke Recovery \& Rehabilitation Research: Issues, Opportunities, and the $\mathrm{NIH}$ Stroke Net. Stroke 2017; 48: 813-19.

13. Munoz-Price LS. Long-term acute care hospitals. Clin Infect Dis 2009; 49: 438-43.

14. Kahn JM, Davis BS, Le TQ, Yabes JG, Chang CCH, Angus DC. Variation in mortality rates after admission to long-term acute care hospitals for ventilator weaning. J Crit Care 2018; 46: 6-12.

15. Jaeger JM, Littlewood KA, Durbin CG Jr. The role of tracheostomy in weaning from mechanical ventilation. Respir Care 2002; 47: 469-80.

16. Chatterjee A, Chen M, Gialdini G, et al. Trends in tracheostomy after stroke: analysis of the 1994 to 2013 national inpatient sample. Neurohospitalist 2018; 8: 171-6.

17. Turcotte LA, Perlman CM, Fries BE, Hirdes JP. Clinical predictors of protracted length of stay in Ontario Complex Continuing Care Hospitals. BMC Health Serv Res 2019; 19: 218.

18. McClave SA, Martindale RG, Rice TW, Heyland DK. Feeding the critically ill patient. Crit Care Med 2014; 42: 2600-10.

19. McClave SA, Heyland DK. The physiologic response and associated clinical benefits from provision of early enteral nutrition. Nutr Clin Pract 2009; 24: 305-15.

20.Gungabissoon U, Hacquoil K, Bains C, Irizarry M, Dukes G, Williamson R. Prevalence, risk factors, clinical consequences, and treatment of enteral feed intolerance during critical illness. J Parenter Enteral Nutr 2015; 39: 441-8.

21. Kutsogiannis J, Alberda C, Gramlich L, et al. Early use of supplemental parenteral nutrition in critically ill patients: results of an international multicenter observational study. Crit Care Med 2011; 39: 2691-9.
22. Cahill NE, Murch L, Jeejeebhoy K, et al. When early enteral feeding is not possible in critically ill patients: results of a multicenter observational study. JPEN J Parenter Enteral Nutr 2011; 35: 160-8.

23. Umscheid CA, Mitchell MD, Doshi JA, Agarwal R, Williams K, Brennan PJ. Estimating the proportion of healthcare-associated infections that are reasonably preventable and the related mortality and costs. Infect Control Hosp Epidemiol 2011; 32: 101-14.

24. Bogason E, Morrison K, Zalatimo O, et al. Urinary Tract Infections in Hospitalized Ischemic Stroke Patients: Source and Impact on Outcome. Cureus 2017; 9: e1014. 